\title{
Response of creatine kinase and lactate dehydrogenase enzymes to rest interval between sets and set-repetition configuration during bouts of eccentric exercise
}

\author{
HOSSEIN MOHAMMADI $^{1, *}$, MOHAMMAD ESMAIEL AFZALPOUR ${ }^{1}$, \\ SEYED HOSSEIN ABTAHI IEVARY ${ }^{2}$
}

\author{
${ }^{\mathrm{I}}$ Department of Sport Physiology, University of Birjand, Birjand, I.R. Iran \\ ${ }^{2}$ Medical Department, University of Gonabad, Gonabad, I.R. Iran \\ ${ }^{*}$ Corresponding author: Hossein Mohammadi; Department of Sport Physiology, University of Birjand, No. 22, Nur Street, \\ Neyshabur city, Razavi Khorasan Province, Birjand 9318653144, I.R. Iran; Phone: +98 9157131382; E-mail: sea_65000@yahoo.com
}

(Received: December 22, 2017; Accepted: January 23, 2018)

\begin{abstract}
Background and aims: Various studies have demonstrated that different rest intervals and set-repetition have a significant effect on hormonal and metabolic responses. These factors can lead to different muscle damage responses. Methods: Forty untrained subjects $(25.4 \pm 0.068$ years, height: $1.74 \pm 0.97 \mathrm{~cm}$, and weight: $30.8 \pm 8.48 \mathrm{~kg}$ ) in three sessions of eccentric resistance exercise with 24 -h rest between each session participated in this study. Subjects were divided into four groups of 10 subjects who performed 50 eccentric contractions with different number of 5 and 10 sets, 5 and 10 repetitions, and the interset rest interval 1 and $3 \mathrm{~min}$ with $85 \%$ of one repetition maximum (1RM). Creatine kinase and lactate dehydrogenase were measured immediately before each session, immediately after each session, and $24 \mathrm{~h}$ after the last training session. Variance analysis with repeated measurement and Bonferroni post hoc test were used for statistical analysis of data. Results: There is no significant difference in creatine kinase and lactate dehydrogenase between four groups at different time points $(p<0.05)$. Conclusion: The repetition of eccentric exercise for three consecutive days causes muscle damage that is independent of manipulating the interset rest intervals and the number of set-repetition.
\end{abstract}

Keywords: eccentric exercise, creatine kinase, lactate dehydrogenase, muscle damage, rest interval

\section{Introduction}

Participation in unaccustomed, eccentrically biased exercise often results in ultrastructural damage to skeletal muscle [1-3]. Symptoms of this exercise-induced muscle damage include elevated muscle proteins in the blood, delayed onset muscle soreness, swelling, a decreased range of motion, and impairment of proprioceptive function and neuromuscular control (for a review, see [4]). One of the consequences of the resistance exercises is the delayed onset muscle soreness. Delayed muscle soreness causes pain, swelling, decreased range of motion, and functional strength reduction [5]. Eccentric contractions can cause muscle pain. To reduce the muscle pain induced by eccentric contractions can be used in practice, which is not possible for most of the sports; also, due to reduced fitness in the off-season, athletes, to do preseason preparation, need to repeat the same exercise intensely and continuously within a few days, and this has caused a lot of athletes and ordinary people to continue to practice while they are having muscle soreness. It is very important to prevent such damage. Since these injuries are inevitable, providing a treatment to help relieve the discomfort, injury, and disability and fast return of the individual to exercise is very important [6]. Warm up before exercise, cool down, and stretch afterward have been introduced as guidelines for the prevention of delayed onset muscle soreness [7].

This is an open-access article distributed under the terms of the Creative Commons Attribution-NonCommercial 4.0 International License, which permits unrestricted use, distribution, and reproduction in any medium for non-commercial purposes, provided the original author and source are credited, a link to the CC License is provided, and changes - if any - are indicated. 
In addition, these methods of treatment to reduce the signs and symptoms of delayed onset muscle soreness have been used. Medications due to their side effects and the possibility of getting their doping agents cannot be an appropriate treatment [8]. Non-drug treatments including massage, ice massage, and ultrasound waves repeated this practice causing subcutaneous nerve stimulation $[9,10]$. But since the main cause and mechanism of this phenomenon is exactly not known, the results of the application of different methods can be different [11].

Repeating this exercise as a method of treatment has been studied on delayed onset muscle soreness. The researchers repeated the research on the effects of exercise and have come to the conclusion that the effect of repeated eccentric exercise is similar to the first, markers of muscle damage, primarily due to reduced exercise. Repeating the same exercise, the researchers concluded that in the first 3 days, due to a series of adaptations in muscle, injury does not cause deterioration of muscle function $[6,10]$. According to studies and eyewitness accounts, team and individual sportsmen in our country have to practice at least three times a week. As the muscle after $48 \mathrm{~h}$ of rest is not completely returned to the initial state, it is not yet clear whether the exercise or performance will decrease in such a situation.

Rodrigues et al. [12] investigated the changes in cellular injury indices after two eccentric exercises with a 4 -week interval with $100 \%$ intensity of one maximum repetition with a difference in contraction. The results of this study showed that the number of contractions versus shiftrepetition manipulation is more effective than muscle damage. Mayhew et al. [13] reported that creatine kinase enzyme activity was more than a 3-min interval after a resistance exercise with an intensity of $65 \%$, a maximum repetition of 10 sets with 10 repetitions, and a 1 -min resting distance. On the other hand, Rodrigues et al. [12] did not show any significant difference in creatine kinase and lactate dehydrogenase enzyme activity after 3 sets and 10 repetitions. Given that even if the total number of eccentric contractions in a similar exercise is similar, the magnitude of muscle damage is likely to vary with the simultaneous manipulation of the number of sets, the number of repetitions, and the rest time between the exercise times, and given that most past research has looked at an outsourcing exercise that is less like an athlete training program; it is a question of which rest time ( $1-3 \mathrm{~min}$ ) and how many times and again it leads to less muscle damage?

\section{Methods}

A total of 40 subjects were selected randomly and divided into four groups of 10: the first group was resting for 1 min and 5 sets with 10 repetitions; the second group was resting for $1 \mathrm{~min}$ and 10 sets with 5 repetitions; the third group was resting for $3 \mathrm{~min}$ and 5 sets with 10 repetitions; and the fourth group was resting for $3 \mathrm{~min}$ and 10 sets with 5 repetitions.

In this study, delayed onset muscle pain free weights (dumbbells) were used. Because, eccentric contractions may cause delayed muscle aches, lifting the second phase, which involves reducing weight with the non-dominant hand was used [10]. To become familiar with the test and one repetition maximum (1RM), subjects were recruited through questionnaires $48 \mathrm{~h}$ before the start of the performance and were invited to the Physical Education School Hall. During their meeting, they started to warm up for $5 \mathrm{~min}$.

After warming up, the estimated 1RM, with 3-5 repetitions and the maximum concentric contraction, was in the non-dominant hand [14]. About $48 \mathrm{~h}$ after the test, measurements were performed in the period between 8 and $11 \mathrm{pm}$. Subjects initially completed the questionnaire and the consent form.

Questions were as follows: Participants should not experience neurological, muscle, heart, and brain disease, and the history of the non-dominant upper limb fractures were included. During the 6 months prior to the study, the non-dominant upper body weighttraining had drug injection and during the 10 days prior to study, it is better for them to feel at the beginning of the training. They did not have high-fat meal, especially during the protocol and the night before each workout had to begin. Height, weight, and blood samples for determination of creatine kinase and lactate dehydrogenase levels were measured.

For the measurement of creatine kinase and lactate dehydrogenase levels, $5 \mathrm{ml}$ of venous blood radius (antecubital) was performed by an expert. Blood samples of $5 \mathrm{~min}$ at room temperature were immediately centrifuged. When the serum was separated, it was stored at $-20^{\circ} \mathrm{C}$ for subsequent analysis (Pars Azmoon kit, Iran). All measurements were performed by one person. The subjects sat on a chair and performed 50 eccentric contractions of $85 \% 1 \mathrm{RM}$ in four groups. Each set includes 10 or 5 contractions that lower a person's weight in $3 \mathrm{~s}$ and at least $2 \mathrm{~s}$ to reach the next contraction without weights placed in full flexion [10]. Around 1- or 3-min rest was given between each set. The experimental protocol was repeated for three consecutive days. All measurements except contusion, immediately before and after each training session, were performed.

\section{Results}

The result of analysis of variance (ANOVA) test showed that the mean of creatine kinase in the four groups was not significantly different at different stages of 
measurement $(p=0.67)$. Also, there is no significant difference between the mean of creatine kinase in the four groups $(p=0.735)$ and the interaction between time and group is not significant $(p=0.329)$ (Table I).

The result of ANOVA showed that the mean of lactate dehydrogenase in the four groups of study was not significantly different in at least two stages of the different stages of measurement $(p=0.60)$. Also, there is no significant difference between mean of lactate dehydrogenase in the four groups $(p=0.901)$ and the interaction between time and the group is not significant $(p=0.164)$ (Table II).

\section{Discussion}

There was no significant difference in the amount of creatine kinase in all stages of time and between different groups by manipulating the resting intervals and the number of sets-repetitions. The results of this study are in agreement with the results of Howatson et al. [15] and Chan et al. [16] and are not consistent with the results of Chen and Hsieh [17] and Mayhew et al. [13]. According to Schwan's theory, the rupture and instability of muscle cell membranes result in the release of intracellular contents into intercostal water and ultimately of plasma, which is one of the possible mechanisms for increasing creatine kinase after eccentric exercises. The amount of creatine kinase increases $24 \mathrm{~h}$ after the eccentric exercise, which gradually reaches its maximum in $48 \mathrm{~h}$ and returns to the baseline in 72-96 h [18]. There is no significant difference in the plasma levels of creatine kinase. One of the interesting findings of this study is that there is no significant difference between the different groups with rest periods and the number of different sets. Similarly, the reduction in creatine kinase is in the $1 \times 10 \times 5$ group. After the first stage, the trend was slowing down. Mayhew et al. [13] reported that creatine kinase enzyme activity was more than a 3-min interval after a resistance exercise with an intensity of $65 \%$, a maximum repetition of 10 sets with 10 repetitions, and a 1 -min resting distance. On the other hand, Rodrigues et al. [12] did not show any significant difference in activity of creatine kinase and lactate dehydrogenase enzyme after 3 sets and 10 repetitions. On the other hand, the rate of creatine kinase response depends on (repetition $\times$ sets/times) and type of rest timing caused different reactions [19]. One of the obvious reasons for the results of this study is the study by Mayhew et al. [13], mechanical load, and different muscle mass and type of exercise areas. In this study, the protocol has been repeated for three continuous days, but Mayhew et al. [13] and Willardson [20] took time only 1 day. There is also another possible reason for the subjects' training status, with the current protocol being implemented on untrained people, but previous studies have resisted practitioners [12], so that some kind of compatibility with practice repetition is observed in trained practitioners to be. On the other hand, the reduction of plasma creatine kinase in the $1 \times 10 \times 5$ group, which after the first time showed a decreasing trend, is another interesting finding of this study. The possible mechanisms for reducing creatine kinase can be attributed to the responsiveness of creatine kinase, which is influenced by individual factors and exercise variables (such as genotype and high responsiveness, body composition and gender, mechanical and metabolic stress, workload and amount of resting intervals between set, the speed of movement, the involved muscle group, the age, and the type of muscle, and repetition eccentric exercise) $[15,18,21]$. In general, the significant difference between the different groups in the response to creatine kinase and the reduction of the response of creatine kinase after the first phase of external exercises implies that apart from the resting intervals between the turns and the number of repetitive sets, important variables. There is another that affects the response to muscle damage

Table I Analysis of repeated measurement variance of creatine kinase in four groups

\begin{tabular}{lccccc} 
Source & Type III sum of squares & $d f$ & Mean square & $F$ & $p$ \\
Time & $4,084.680$ & 3.193 & $1,279.449$ & 0.570 & 0.647 \\
Group & $51,736.120$ & 3.000 & $17,245.373$ & 0.427 & 0.735 \\
Interaction & $24,852.480$ & 9.578 & $2,594.856$ & 1.156 & 0.329 \\
\hline
\end{tabular}

Table II Analysis of repeated measurement variance of lactate dehydrogenase in four groups

\begin{tabular}{lclcrc} 
Source & Type III sum of squares & $d f$ & Mean square & $F$ & $p$ \\
Time & $35,076.250$ & 4 & $8,769.062$ & 2.319 & 0.060 \\
Group & $7,005.180$ & 3 & $2,335.060$ & 0.193 & 0.901 \\
Interaction & $64,327.670$ & 12 & $5,360.639$ & 1.418 & 0.164 \\
\hline
\end{tabular}


indices. On the other hand, there was no significant difference in the level of lactate dehydrogenase at any stage of time and also among all groups. Lactate dehydrogenase increased after the first time and showed a decreasing trend after the last training. In $24 \mathrm{~h}$ after the last exercise, in the four groups, it showed an increasing trend. The results of this research are consistent with the results of Chen et al.'s research [17] and are not consistent with the results of the researches by Arazi and Rahimi [19] and Mayhew et al. [13].

Possible mechanism of increasing lactate dehydrogenase after mechanical stress (decomposing sarcomere and components of $z$ lines) and metabolic pathways (increasing the permeability of the membrane of the cell and thereby increasing the calcium ion) activates the potassium channels and protein-degrading enzymes. However, the reduction of lactate dehydrogenase after the first time, after the last one, which is one of the interesting findings of this study and contradictory with [13] and [19], is due to the fact that the response of lactate dehydrogenase such as creatine kinase is influenced by duration of exercise, nutrition and supplements, the number of movements, and muscle groups involved $[12,16,18]$.

\section{Conclusions}

In general, the results of this study showed that repetition of eccentric exercise during three consecutive days increased muscle damage and did not result in adaptation, which is independent of the difference in rest intervals between sets and number of set-repetitions configuration.

Funding sources: The research received no specific grant from any funding agency in the public, commercial, or not-for-profit sectors.

Authors' contribution: HM, MEA, and SHAI performed and designed experiments, prepared figures, and revised the manuscript. All authors read and approved the final manuscript.

Conflict of interest: None.

\section{References}

1. Fridén J, Lieber RL: Eccentric exercise-induced injuries to contractile and cytoskeletal muscle fibre components. Acta Physiol Scand 171, 321-326 (2001)

2. Maughan RJ, Donnelly AE, Gleeson M, Whiting PH, Walker KA, Clough PJ: Delayed-onset muscle damage and lipid peroxidation in man after a downhill run. Muscle Nerve 12, 332-336 (1989)
3. Fridén J, Sjöström M, Ekblom B: Myofibrillar damage following intense eccentric exercise in man. Int J Sports Med 4, 170-176 (1983)

4. Byrne C, Twist C, Eston R: Neuromuscular function after exerciseinduced muscle damage: Theoretical and applied implications. Sports Med 34, 49-69 (2004)

5. DiPasquale DM, Bloch RJ, Lovering RM: Determinants of the repeated bout effect following lengthening contractions. Am J Phys Med Rehabil 90, 816-824 (2011)

6. Moradi L: Effect of subcutaneous nerve stimulation and massage on delayed onset muscle soreness of functional markers for the students of Tehran. Faculty of Physical Education, Master's Thesis, School of Physical Education, Central Tehran Branch, Tehran (2002)

7. Nosaka K, Sakamoto K, Newton M, Sacco P: How long does the protective effect on eccentric exercise induce muscle damage last? Med Sci Sports Exerc 33, 1490-1495 (2001)

8. Rodenburg JB, Steenbeek D, Schiereck P, Bär PR: Warm-up, stretching and massage diminish harmful effects of eccentric exercise. Int J Sports Med 15, 414-419 (1994)

9. Howatson G, Gaze D, van Someren KA: The efficacy of ice massage in the treatment of exercise-induced muscle damage. Scand J Med Sci Sports 15, 416-422 (2005)

10. Nosaka K, Clarkson PM: Muscle damage following repeated bouts of high force eccentric exercise. Med Sci Sports Exerc 27, 12631269 (1995)

11. Cleary MA, Kimura IF, Sitler MR, Kendrick ZV: Temporal pattern of the repeated bout effect of eccentric exercise on delayed onset muscle soreness. J Athl Train 37, 32-36 (2002)

12. Rodrigues BM, Dantas E, Salles BF, Miranda H, Koch AJ, Willardson JM: Creatine kinase and lactate dehydrogenase responses after upper body resistance exercise with different rest intervals. J Strength Cond Res 24, 1657-1662 (2010)

13. Mayhew D, Thyfault J, Koch A: Rest-interval length affects leukocyte levels during heavy resistance exercise. J Strength Cond Res 19, 16-22 (2005)

14. Shahbazpour N, Carroll TJ, Riek S, Carson RG: Early alterations in serum creatine kinase and total cholesterol following high intensity eccentric muscle actions. J Sports Med Phys Fitness 44, 193-199 (2004)

15. Howatson K, Van Someren K, Hortobágyi T: Repeated bout effect after maximal eccentric exercise. Int J Sports Med 28, 557-563 (2007)

16. Chan R, Newton M, Nosaka K: Effects of set-repetition configuration in eccentric exercise on muscle damage and the repeated bout effect. Eur J Appl Physiol 7, 2653-2661 (2011)

17. Chen TC, Hsieh SS: Effects of a 7-days eccentric training period on muscle damage and inflammation. Med Sci Sports Exerc 33, 1732-1738 (2001)

18. Mohammadi H, Sahebazamani M, Ghahraman Tabrizi K: The effect of repeated bouts of eccentric exercise on some of biochemical markers of delayed onset muscle soreness. Int J Appl Exerc Physiol 4, 34-39 (2015)

19. Arazi H, Rahimi R: The effect of different rest intervals between multiple bench press bouts. S Afr J Res Sport Phys Educ Recreation $33,1-8$ (2011)

20. Willardson JM: A brief review: Factors affecting the length of the rest interval between resistance exercise sets. J Strength Cond Res 20, 978-984 (2006)

21. Pereira R, Machado M: Resistance exercise- induced microinjuries do not depend on 1 or 3 minutes rest time interval between series. Int J Sci 4, 1885-3137 (2007) 